\title{
Heard but not seen: Exploring youth counsellors' experiences of telephone counselling
}

\author{
Danielle Davidson, Queensland University of Technology, Queensland, Australia \\ Gai Harrison, Allied Health, Royal Brisbane and Women's Hospital, Queensland, Australia
}

\begin{abstract}
INTRODUCTION: Human service practitioners who work over the telephone are physically invisible to their clients and this invisibility shapes their work. Existing literature suggests that physical invisibility, coupled with anonymity and the immediacy of service provision are defining features of telephone counselling. However, little research has explored how telephone counsellors experience these features in any real depth.
\end{abstract}

METHODOLOGY: This article reports on a case study conducted at a youth helpline in Aotearoa New Zealand. Qualitative, semi-structured interviews were conducted with 10 practitioners on their experiences of telephone counselling work. One key finding from this case study is discussed: the impact of invisibility and anonymity.

FINDINGS: Findings indicate that practitioners' experiences of delivering telephone counselling are more divergent than those presented in extant literature. While telephone counsellors face challenges delivering non-face-to-face counselling support, the physical invisibility of this medium, coupled with a supportive work environment can also provide potential benefits to counsellors.

CONCLUSIONS: Counsellors' experiences of telephone counselling work appear to be more nuanced than traditionally understood. While practitioners may experience a range of possible challenges in delivering telephone counselling, such as responding to hoax and abusive callers, they also experience benefits such feeling relaxed, and supported by colleagues and supervisors. Management practices, such as flexible rules and accessible supervision, can help practitioners manage the impact working non-face-to-face with clients. Given the ongoing popularity of telephone counselling, further research is needed on the working conditions that promote practitioner wellbeing and job satisfaction.

KEYWORDS: Telephone counselling; invisibility; anonymity; telephone counsellor wellbeing; working conditions
AOTEAROA NEW ZEALAND SOCIAL WORK 32(1), 73-85.

CORRESPONDENCE TO: Danielle Davidson danielle.davidson@qut.edu.au 
experience delivering support to clients over the telephone. This is a significant omission given the important role that work plays in shaping practitioner wellbeing, job satisfaction and performance (Shier \& Graham, 2011).

Practitioners may experience challenges performing telephone counselling due to the unique characteristics of this work. These characteristics include features such as its non-face-to-face format and its associated anonymity and invisibility (Christogiorgos, et al., 2010). The physical invisibility of counsellors and clients from each other is a key defining feature of telephone counselling work. While some research has acknowledged these unique characteristics and their impact on counsellors, the major focus of these studies is the impact of these features on callers (Christogiorgos et al., 2010; Coman, Burrows, \& Evans, 2001; Rosenfield, 2003).

This paper reports one of the key findings from a larger doctoral study on counsellors' experiences of telephone counselling work, namely the impact of physical invisibility and client anonymity on practitioners. In this study, telephone counselling refers to a free service that provides both one-off and ongoing support for children and young people aged 5-19 years to talk about their problems and concerns. The main premise of this paper is that telephone counsellors are heard but not seen by their callers, and this feature of their work has nuanced implications for how practitioners experience this work, as well as their wellbeing and job satisfaction.

\section{Telephone counselling in the contemporary social services context}

Counselling as a profession is relatively young in Aotearoa New Zealand.

The provision of counselling services underwent significant transformation in the late 20th century in response to Aotearoa New Zealand shifting from a welfare state to a free market economy (Hermansson \& Webb, 1993; Stanley \& Manthei, 2004). This has led to the outsourcing of counselling to the private and charity sectors, and an increase in user-pays counselling services (Rodgers, 2012). Although the profession initially relied on western theories of counselling, socio-political-cultural developments in the late 1980s and early 1990s saw the call for locally informed counselling theories and approaches (Durie \& Hermansson, 1990; Hermansson \& Webb, 1993; Lang, 2005). This shift in approach encourages counselling services and practitioners to act in the spirit of partnership set out by the Treaty of Waitangi (Miller, 2012). For instance, Durie and Hermansson (1990) called for Te Ao Māori (Māori worldview) concepts to be incorporated into practice, especially when working with Māori clients (Hermansson \& Webb, 1993). There is an obligation for practitioners and services to ensure their work is reflective of multiple local understandings, or bicultural pluralism (Lang \& Gardiner, 2014), and a commitment to culturally competent and safe practice (Rodgers, 2012). However, despite the commitment to biculturalism, Māori-centred practice challenges remain, such as ongoing differences between Māori and Pākehā interpretations of the meaning and intention of Te Ao Māori concepts (Watson, 2019), and the impacts of increasing professionalisation of counselling and social work practice on future practitioners and their practice (Staniforth, 2010).

Research exploring practitioners' experiences of telephone counselling is limited, with most studies carried out prior to 2005. A recent desktop review of extant literature on telephone counselling found the current emphasis is on its effectiveness in responding to specific presentations, such as smoking or alcohol and drug use, rather than general mental health support. Within the Aotearoa New Zealand context, a small body of research has examined the medium's effectiveness in supporting young 
people (Youthline, 2005) and adults who gamble (Kim, Hodgins, Bellringer, \& Abbott, 2016), and its role in supporting people from culturally and linguistically diverse (CALD) communities with mental health issues (Dong, 2016). However, limited research has been conducted on how counsellors and other human service practitioners experience this work.

Studies in Australia and the UK investigating the use of telephones as a medium of human service delivery (e.g, child protection helplines) have examined the impact of providing appropriate support for people with complex presentations over the telephone (King, Nurcombe, Bickman, Hides, \& Reid, 2003; van den Broek, 2008). Relative to practitioner experiences, this research highlights workers' concerns over increased surveillance by management, and frustrations over how this can limit the professional discretion needed to respond effectively to callers (van den Broek, 2008). While critical of the tendency for callcentre mediums to constrain professional autonomy and discretion, these studies acknowledge the important role they play in providing accessible and timely support to service users. In other words, they provide immediate, and usually free, support at the point of crisis and are accessible to those who are socially and geographically isolated (Coman et al., 2001). Accordingly, Parrott and Madoc-Jones (2008) argue that, rather than just viewing information and communication technologies (ICT) as tools of managerialism, it is important to recognise their potential to reach disenfranchised and excluded service users. This body of literature, while patchy in its depth and breadth, demonstrates the important role telephone support services play in the contemporary human services landscape.

\section{The invisible telephone counsellor and its impacts}

Physical invisibility, or the lack of visual cues such as facial expression and body language, is a defining feature of telephone counselling. (O'Sullivan \& Whelan, 2011). Telephone practitioners form their connection with service users audiologically (Maher \& von Hippel, 2005). This style of interaction requires practitioners to adjust their communication style and concentrate on other facets of the caller's presentation apart from visual cues (Christogiorgos et al., 2010). Coman and colleagues (2001, p. 254) argue that telephone counsellors need to gain competence in reading "every sound, every silence, inflection and qualities of speech including tone, pitch and speed" in order to determine what is happening for the caller. In addition, they need to be aware of their own speech properties, or paralanguage, including their "speaking rate, tone, volume, pitch and intonation" (Hanna \& Nash, 2012 p. 486), and what this might convey to the person at the other end of the telephone line. Finally, telephone counsellors need to be skilled at interpreting and interrupting silences to communicate presence and ensure the therapeutic relationship with the caller remains intact (Sanders, 2007).

The physical invisibility and associated anonymity of callers provides service users with a degree of control over the counselling process (Rosenfield, 2003). In comparison to face-to-face services, callers have greater ownership over the counselling process as they are in charge of when, or if, to ring up, where to call from, how much to disclose, and the duration of the call (Christogiorgos et al., 2010; Rosenfield, 2003). The ability to remain anonymous may also promote the ease of caller self-disclosure (Centore \& Milacci, 2008). Suler (2004) argues that, in non-face-to-face environments, people experience a sense of benign disinhibition as they feel less inhibited and freer to share information than they would in face-to-face settings. This aligns with research that has found clients experience greater freedom in expressing their presenting concerns and feelings over the telephone (Coman et al., 2001; Rosenfield, 2002). The lack of identifying information both parties have about each other is believed to provide a sense of safety and security for the caller and 
promote heightened intimacy between the counsellor and the caller (Christogiorgos et al., 2010). Accordingly, callers have identified the ability to remain anonymous as one of telephone counselling's most attractive features (Bobevski, Holgate, \& McLeannan, 1997).

While anonymity affords benefits to callers, it may deliver negative outcomes for counsellors. Callers have been reported to contact telephone services to abuse and / or yell at a counsellor (Lester, Brockopp, \& Blum, 2012). Suler (2004) calls this toxic disinhibition, where the physical distance and non-face-to-face interaction may encourage inappropriate behaviour, such as pretending to be someone else or expressing threats towards others. Toxic disinhibition occurs when callers present by 'testing' services in ways such as abusing counsellors or calling numerous times within a short period of time (Hall \& Schlosar, 1995; Sanders, 2007). The counsellor's ability to manage these presentations may be further complicated by organisational directives, with many services adopting a strong 'no hang up' philosophy for testing calls (Hall \& Schlosar, 1995; Sanders, 2007). Ongoing engagement with testing callers may lead to counsellors feeling uncomfortable, distressed or violated, in turn promoting a sense of powerlessness (Sharah, 1995). As a result, several authors suggest that over time, responding to testing calls may negatively impact counsellors' attitudes towards all types of callers (Hall \& Schlosar, 1995; Lester et al., 2012).

Despite the acknowledgement of the unique characteristics of telephone counselling work, little research has explored how practitioners' experiences are shaped by such features. Thus, this paper will explore how practitioners' experiences are shaped by the invisibility and anonymity of telephone counselling work through examining the experiences of one group of counsellors working at a youth helpline in Aotearoa New Zealand. In doing so, it will address the following research question:
How do unique work characteristics of telephone counselling, namely invisibility and anonymity, shape the experiences of telephone counsellors?

\section{Methodology}

The broad aim of the study was to explore how youth counsellors experience telephone counselling, and the role work-related factors played in shaping these experiences. The findings reported in this paper stem from a qualitative case study of youth counsellors' experiences of working in an Aotearoa New Zealand youth telephone counselling service. The service provides free counselling to children and young people between 6 and 18 years of age. Prior to conducting the study, approval was gained from the Human Research Ethics Committee at The University of Queensland.

\section{Research design}

Multiple sources of data were collected for this case study; however, this paper reports only on findings from semi-structured interviews that focus specifically on how invisibility and anonymity shape practitioner experiences of telephone counselling work. Holstein and Gubrium (2004) argue that interviewing is an active process whereby both the interviewer and participant create meaning through their conversation. By acknowledging the active nature of in-depth interviewing, the researcher was conscious of the freedom participants had to construct their experiences through dialogue and the role the researcher played in co-construction of these experiences.

An interview guide was developed, which listed the main topics to be explored (Minichiello, Aroni, \& Hays, 2008). This provided flexibility in when and how topics were explored, while ensuring the main topics were covered to address the central research questions (Alston \& Bowles, 2018; Patton, 2002). The topics covered included: 1) professional background and length of telephone counselling experience; 
2) perceptions and experiences of telephone counselling; and 3) the work-related factors that facilitated these experiences.

\section{Participants and recruitment}

Participants consisted of 10 out of the 15 telephone counsellors employed by the youth telephone counselling service. They were aged between 21 and 50 years, with most having social service qualifications such as in psychology, counselling, social work or human services. To ensure participants had an adequate range of experiences to reflect upon, only those who had worked as a telephone counsellor for a minimum of 12 months were eligible to participate. Participants were introduced to the study through the service's Chief Executive Officer (CEO). Four participants contacted the researcher about their interest in participating in the study prior to the first author's arrival at the research site. An additional six participants were recruited in person with the assistance of a shift supervisor and conducted in a manner to ensure voluntary participation in the study. Prior to the study, interested participants were informed of what their participation would involve and its voluntary nature, and provided consent based on this understanding.

\section{Data analysis}

Braun and Clarke's (2006) framework for thematic analysis was applied to structure the analysis of interview data. They argue that thematic analysis has been criticised for the lack of clarity around how it should be conducted. To address this reported weakness, they outline six key steps to guide the process of thematic analysis. An additional attraction of this framework is its adaptability to the unique qualities of the data being analysed.

The analysis process began with the transcription of digitally recorded deidentified interviews, which were then transferred into NVivo computer software for data management and coding. Initial coding was conducted using sensitising concepts drawn from extant empirical and theoretical literature to explore how the counsellors' experiences resonated with themes and concepts in this literature (e.g., the unique characteristics of this work) (Patton, 2002). Next, data-driven coding was completed to identify unique themes that emerged from participants' accounts of their work (Ryan \& Bernard, 2003). The codes were then refined into overarching themes that were defined and named accordingly (Braun \& Clarke, 2006). The rationale for choosing and identifying themes was made as transparent as possible through writing memos, keeping a reflexive journal, and attaching notes within NVivo (Maxwell, 2005). The anonymity of participants was ensured by omitting content that may be personally identifying, which was especially important due to the size of the service.

To help to minimise the impact of researcher bias, the lead author engaged in a process of ongoing self-reflection, or reflexivity, during data collection, data analysis and the write-up of findings. This involved keeping a journal documenting her experiences, reactions, emerging understandings and interpretations, and possible impact on the research at each stage of the investigation process. The self-reflective process of keeping a reflective journal heightens researchers' awareness whether their own experiences were influencing the perception and construction of participant experiences (Altheide \& Johnson, 1994), thus enhancing the credibility of the findings (Lincoln \& Guba, 1985).

\section{Findings}

This section examines the findings in relation to four key themes related to the invisibility and anonymity of telephone counselling work, focusing on the implications for human service practice via this medium. The first theme focuses on responding to challenging caller presentations; fostered by the anonymity of the service, counsellors 
identified these presentations to have a considerable impact on their work. The positive aspects of invisibility are then exemplified through the following three themes: enhanced sense of freedom and physical comfort; the benefit of being able to see and interact with colleagues; and the accessibility of supervision.

\section{The impact of responding to challenging caller presentations}

Similar to Hall and Schlosar (1995) and Sanders (2007), this study found that the physical invisibility and the anonymity of telephone counselling led to conditions where callers felt free to 'test' out the service and its counsellors. This section outlines the impact such 'testing' calls had on counsellors in relation to a growing scepticism of the genuineness of callers, and a waning desire to work with such calls.

'Testing' calls were found to create extra challenges for counsellors in establishing therapeutic or working relationships with service users over the telephone, and maintaining the energy needed to respond appropriately. Abusive calls were experienced as especially difficult. The majority of counsellors indicated that ongoing exposure to testing calls during their shifts was tiring and de-motivating. This is illustrated by Participant 5 who reflected on the impact of these testing calls over the course of her telephone counselling career:

When we had our introduction into these things you know you have to be prepared to deal with this, I was thinking of course I can deal with this, but it's quite tiring...I'm feeling my patience comes to an end... Yeah I think I'm a bit levelling out, because all the enthusiasm from the beginning is gone and sometimes it's just really hard and frustrating to go through so many testing calls.

Other participants reported an increase in scepticism with regard to the genuineness of caller presentations:
It's not easy and what I find is the longer you do it...is that you become a little bit cynical. So initially I was surprised when it was a testing [call], now it's the opposite. I'm sort of geared, always listening for a testing call, listening for the inconsistencies...I have to confess I think on one or two occasions I've gone into the call absolutely convinced it was a testing, and it's taken me a good ten minutes into the call to suddenly realise, hey this is a really serious call. So from that way, that sort of carries a little bit of guilt with it. (Participant 10)

Hall and Schlosar (1995) observe that repeated interactions with testing callers can diminish counsellors' empathy and result in an expectation of being manipulated by callers. Hence it is not surprising that three participants in this study questioned the overall value of responding to testing calls.

Ambiguous presentations, calls where the telephone counsellors did not know whether the caller was legitimate or a hoax, were also experienced as challenging. In these cases, counsellors found it difficult to believe in the genuineness of callers' presentations, at least at the start of the call. This is highlighted by Participant 2:

Like when it's clearly adult and...they're trying to be...young. Or if it's a child pretending, saying something really, really, really serious and I can tell that... they're just fooling around. But it's over the phone I have to go by... whatever the person was saying...So, it can be quite challenging.

Although she did not always believe a caller's presentation, Participant 2 felt unable to respond in any way other than to go along with the presentation because she lacked visible cues to confirm her suspicions.

Workers' difficulties experienced in responding to testing calls is partly explained by the organisational expectation of treating each call as though it is the caller's 
first contact with the service. As Participant 10 reflected:

I think there's this sense of being a little bit powerless with that, instead of going okay you have phone[d] six or seven times and I don't want to talk to you anymore, we sort of feel like you are having to buy into it even though you know...they're lying.

As a result, this participant felt unable to challenge callers' presentations, and felt obliged to go along with fictitious presentations, illustrating the lack of control counsellors may have over hoax callers. Ultimately, testing or hoax callers impacted counsellors' willingness to build a relationship and maintain a connection with callers, demonstrating the difficulty of adhering to the organisational directive to treat each caller as though it was their first call.

While responding to ambiguous or testing calls is challenging and may engender a sense of powerlessness, participants also identified positive aspects of being invisible to callers which are explicated in the following three themes.

\section{Enhanced sense of freedom and physical comfort}

Participants reported that the invisibility of callers promoted a sense of freedom and ease whilst counselling over the telephone. Two counsellors described how not being able to see callers allowed them to relax and attend to their own physical comfort when interacting with callers:

Often, I'll take my shoes off, my feet up, yeah that kind of stuff, 'cause it makes me feel more comfortable, or sit on my feet on the chair, like you know on my knees. (Participant 8)

I kind of like to sit weirdly, like cross my legs on my chair, or bring my knees up. [Laughs] You couldn't really do that when you are with a client [face-to-face]. (Participant 1)

These counsellors surmised that they have greater freedom of physical expression when counselling via the telephone than in face-to-face settings. This freedom extended to what they wore to work, with Participant 1 commenting that they may even dress in "tracksuit pants and slippers" if they chose.

Over time, counsellors learnt there was a degree of flexibility around the organisational rules and behavioural expectations, facilitating a perceived degree of freedom when working in the counselling space. For example, counsellors were cautioned against putting their feet up on their desks, playing solitaire or searching on the Internet whilst on a call, and talking to fellow counsellors in between calls. However, the participants in this study reported that they continued to engage in these activities despite the service's expectation that these behaviours should be avoided. This ability to break some rules - and get away with it - may be indicative of an organisational culture that privileges the counsellors' needs and preferences over formalised conventions.

The counsellors rationalised their behaviour by suggesting that these sorts of activities assisted them to manage various aspects of their work. Five counsellors commented on how these activities distracted them from the challenges of their work and enabled them to relax in the counselling environment. For example, putting their feet on their desks helped them to stay calm while on testing calls. When asked how she handled testing calls one participant commented:

I might be kind of swivelled around on my chair and maybe even my legs up on the desk or something like that...even though we're not allowed to do that. (Participant 1)

In addition, three counsellors commented on how playing solitaire or looking up 
information and other material on the internet helped to distance themselves from repeat testing calls, allowing them to effectively manage the impact of these calls.

...I find, even though we' re not allowed to going on the internet, which pretty much all of us do, but we're not suppose[d] to... It's just that space...and shift in attention. (Participant 8)

Hence, this flexibility in terms of some organisational expectations enabled the telephone counsellors to draw on their own coping strategies to deal with the challenging features of their work.

The benefit of this sense of freedom was expressed by others as enabling them to adopt a more relaxed demeanour when working with callers over the telephone. For instance, Participant 7 stated:

...they [callers] can't see...non-verbal[s] and also just allowing us to be ourselves as well. So it's kind of like we can be open to an extent...but you don't have to be so professional like a face-to-face person, you can be more relaxed.

The invisibility and anonymity of telephone counselling enabled workers to express themselves more freely and helped to facilitate a greater sense of ease when working over the telephone with service users.

\section{The benefit of being able to see and interact with colleagues}

A surprising finding from this study is the benefit participants derived from being able to see and interact with each other in the counselling floor. The counselling floor was set out in an open-plan format, facilitating practitioner ability to see and interact with their colleagues. Most counsellors identified visually and verbally connecting with colleagues as an important means of managing the challenging aspects of their work. Although the management preferred counsellors to talk via the service online hyperchat system the majority preferred talking in person.

...sometimes that type of [in-person]... interaction is better for me...rather than just chatting by messaging, just having that more social interaction. It's a nice break from just having that interaction over the phone. (Participant 2)

Four other counsellors similarly indicated that in-person interactions with their peers were a welcome respite from the nonface-to-face interactions they experienced with callers. Although this in-person communication between counsellors was not the service's preferred means of communication, the participants indicated that they were not reprimanded by supervisors if they chatted this way with their colleagues.

The capacity to interact with colleagues both verbally and visually played a large role in counsellors' self-care. This was seen as an avenue to reduce the isolating nature of the work:

...even though you are around a lot of people, you're not necessarily working with those people, you are working...on your own with the clients and you can become very isolated if you're not careful. (Participant 3)

The close proximity of colleagues in the counselling room and the simple act of being able to see them was identified as an important coping tool:

...it's like a shared place where you can just hang out and it feels more like oh you know we're talking with kids, there's other counsellors here, I'm not working by myself taking all these calls and stressing out. (Participant 7)

Being in a shared space similarly enabled the counsellors to communicate more easily. Several participants commented 
that chatting to peers helped them to pace their calls:

So, it helps the day go by faster being able to have a break...in between calls from our callers, being able to chat to each other. Yeah...I think that's what's... nice it's a good break...from our calls. (Participant 2)

Being surrounded by colleagues also allowed counsellors to 'vent' and offload any frustrations or concerns about their callers. For Participant 2, the ability to readily debrief with colleagues allowed her to move on to the next call unencumbered by residual feelings about the previous caller:

Yeah...it's nice, it helps with...releasing any type of...feelings or stress or anything like that. You know...by venting to someone else, being like, oh my god, that was just so this or that was so that. They will say "yeah I know I had blah, blah, blah"... And then it would be like I'm good now, next call.

The ease with which the counsellors could interact with each other also enabled them to enlist help from colleagues when needed. For example:

...last night...[caller] kept constantly ringing me. So I got him and hung up and then the next person got him. So you just say "this is so annoying" or you know "I can't deal with him do you want to take him?" So it's quite nice to share this... (Participant 5)

The ability to share these types of calls with colleagues reduced the pressure of feeling solely responsible for responding to nuisance callers and suggests that a culture of collegiality was present within the counselling space. In the case of this service, the close proximity and visibility of colleagues not only counteracted the isolating nature of the work but also contributed to a sense of working collectively as a group.

\section{Accessibility of supervision}

The telephone counsellors who participated in this study strongly valued the supervision available to them when on shift. This supervision was accessible to counsellors both during and after calls. Being invisible to callers meant that the counsellors could interact with a shift supervisor online during a call, as identified by Participant 8 .

Well you have instant support at the push of a button... So there's someone that is always there listening if we need them... and to be able to get input if you're struggling. That is really helpful.

The counsellors valued having this immediate access to a supervisor, which appeared to be a significant factor in enabling them to cope with more serious presentations, such as suicidal ideation or child abuse.

Supervision was also reported to be readily available after a call had finished. Most counsellors identified shift supervision as being particularly important because of its accessibility:

...it's fantastic because it's always there.

(Participant 5)

...if I ever have anything I need to talk about or want to talk about there will always be someone who I can talk to. (Participant 4)

The opportunity to debrief with supervisors at regular intervals was valued by participants as a means of processing the content of their shift.

You know for four or five hours you've been on giving your ear and you know that they appreciate it, but in order to keep going you need it [debriefing]. I mean it's the same thing as we say to them "you need to let it out and not carry it" and the same applies for us. (Participant 1) 
This ability to talk with supervisors in a safe and supportive environment when needed gave counsellors the opportunity to express their own thoughts and to be heard. In addition, the quality and accessibility of supervision also contributed to counsellors' reported enjoyment of their work:

I think it's...such an amazing place to work because if ever there's anything going through your mind that you feel like you need to talk about there's, it's [support] just there. (Participant 8)

\section{Discussion}

This paper has examined telephone counsellors' experiences of key features of their work, the physical invisibility and anonymity. While the findings mirror those outlined in previous research demonstrating how invisibility can increase practitioners' vulnerability to receiving abusive and testing calls (Hall \& Schlosar, 1995; Lester et al., 2012), they also illustrate divergent experiences of enhanced subjective wellbeing and work enjoyment. Reported benefits of being invisible to callers included: greater freedom of self-expression both professionally and physically; being able to readily connect with colleagues; and immediate access to supervisory support. Notably, these three factors helped to mitigate the negative impact of responding to challenging testing calls and afforded practitioners a sense of safety and support.

Two key organisational factors appear to have encouraged favourable practitioner experiences of telephone counselling work: flexible organisational rules, and accessible supervision. First, flexible organisational rules facilitated a sense of physical and professional freedom when working with clients over the telephone and facilitated practitioners' capacity to debrief with and receive support from colleagues. Callcentres are traditionally reputed to be places characterised by control over, and compliance by, workers, which are reported to inhibit worker control and discretion (van den Broek, 2008). In addition, employee communication with colleagues is reportedly discouraged in call-centres due to the perception such interactions distract from the core task of answering calls (Barnes, 2007; van den Broek, 2008). However, as observed in this study, flexible and supportive management practices can strengthen practitioner capacity to manage and work with challenging caller presentations and enhance the ability to draw on their own coping strategies to deal with the challenging features of telephone counselling work. This aligns with Hall and Schlosar (1995) who argued that giving telephone counsellors some discretion in dealing with testing calls helps to maintain their morale and work quality.

Flexible organisational rules around how counsellors physically presented themselves, in both clothing and body posture, and how they communicated with colleagues fostered an environment where they felt free to authentically express themselves. Christogiorgos et al., (2010) argues that freedom of expression can help to strengthen connections between counsellors and callers. In this study, the greater sense of freedom reported by participants appears to facilitate adoption of a professional demeanour that is relaxed and at ease. This may have knock-on benefits for callers in terms of availability and responsivity of practitioners, with these features having been found to improve the ease of developing a therapeutic alliance with clients and encouraging positive outcomes (Harms, 2015).

A second key organisational factor was the provision of accessible supervision when practitioners needed it. In this way, practitioners were never left on their own to cope with a challenging call or its after-affects. The strength of this accessible supervision model was that counsellors were able to work on the problems and concerns that were bothering them in the here and now. Kinzel and Nanson (2000) suggest the ability to debrief in this way provides practitioners with the opportunity to externalise concerns, 
thereby accelerating recovery and reducing the impact of calls on their wellbeing. In addition, it provides further evidence of the valuable role internal supervision can play in promoting work wellbeing and perception of management support when it balances organisational responsibilities with the opportunities for reflective supervision (Carpenter, Webb, \& Bostock, 2013; Holz, 2019). While some studies have indicated that call centres may heighten the perception of being monitored by management (Hanna, 2010), if the supervision provided is supportive versus coercive it may enhance counsellors' capacity to manage the demanding nature of their work. Such supportive versus managerial supervisory practice aligns with the goals of cultural supervision (Beddoe, 2016) by providing practitioners with a safe space to reflect on practice issues. However, further research is needed on how to best embed Te Ao Māori concepts in supervision within the telephone counselling context. Due to the managerial environment of telephone counselling services (van den Broek, 2008) it is important to understand how cultural supervision can be enacted within these contexts.

In the current climate of managerialism, which has negatively impacted workers' experiences, for instance through workintensification processes and restrictions on professional autonomy (Fraser \& Taylor, 2016), any improvement in worker conditions that has no negative implications for clients is to be welcomed. Rhoades and Eisenberger (2002) suggest that a perception of organisational support positively influences organisational commitment, job satisfaction, mood, job involvement and interest in work, work performance, and the desire to stay with an organisation. In addition, they suggest it reduces negative psychological and psychosomatic reactions to work, and withdrawal behaviour. Thus, the perceived organisational support present in a telephone counselling service can play an important role in offsetting the negative impacts fostered by the invisibility of this work. Whilst the findings from this study are not generalisable, Stake (2005) argues that case study research can provide important insights into the research phenomenon and may resonate with practitioners working in similar contexts.

Future research is needed to extend understanding about the role workrelated factors play in shaping telephone counsellors' experiences and wellbeing outcomes. A limitation of the reported study is that we did not explicitly investigate Māori practitioner experiences of telephone counselling work. An important area for future research is to explore how Māori practitioners experience working via this medium and how this context impacts upon their interactions with both Māori and Pākehā clients. For instance, how do Māori practitioners overcome the preference for kanohi ki te kanohi, honest and genuine face-to-face interactions (Roberts, 2016). Do Māori practitioners also experience a heightened sense of physical freedom and relaxed professional demeanour over the telephone and, if so, does this enhance their ability to enact manaakitanga, demonstrating care for someone through compassion and embracing of a 'down-to-earth' professional style (Durie \& Hermansson, 1990)? Such research would help to enhance understanding of how Māori practitioners can be supported to work in a culturally responsive way over the telephone.

\section{Conclusion}

Overall, the findings discussed here suggest that the impact of invisibility on counsellors' experience of their work is more nuanced than previously understood. This study has illustrated the key role that organisational and collegial support can play in assisting practitioners to manage the complexities of telephone counselling work. Being a small study based on one service, it is not possible to extrapolate the findings to other settings. However, the study is noteworthy because it contrasts with previous studies which have predominantly depicted telephone counselling in a negative light. Moreover, 
it points to a need for further research on the working conditions and factors that sustain telephone counsellors rather than predominantly focusing on the factors that promote negative outcomes such as workplace stress and job turnover. Finally, further research on Māori practitioner experiences of working in a non-face-to-face context is required to better understand how the limitations of this medium can be addressed to ensure authentic culturally responsive practice is enacted via this medium.

Accepted: 20 February 2020

Published: 14 April 2020

\section{References}

Alston, M., \& Bowles, W. (2018). Research for social workers: An introduction to methods. (4th ed.). Sydney, NSW: Allen \& Unwin.

Altheide, D. L., \& Johnson, J. M. (1994). Criteria for assessing interpretive validity in qualitative research In N. K. Denzin \& Y. S. Lincoln (Eds.), Handbook of qualitative research, (pp. 485-499). Thousand Oaks, CA: Sage Publications.

Barnes, A. (2007). The construction of control: The physical environment and the development of resistance and accommodation within call centres. New Technology, Work and Employment 22(3), 246-259. doi:10.1111/ j.1468-005X.2007.00197.x

Beddoe, L. (2016). Supervision in social work in Aotearoa New Zealand: Challenges in changing contexts. The Clinical Supervisor, 35(2), 156-174. doi:10.1080/073252 23.2016.1217497

Bobevski, I., Holgate, A. M., \& McLennan, J. (1997). Characteristics of effective telephone counselling skills. British Journal of Guidance \& Counselling, 25(2), 239-249.

Braun, V., \& Clarke, V. (2006). Using thematic analysis in psychology. Qualitative Research in Psychology, 3(2), 77-101.

Carpenter, J., Webb, C. M., \& Bostock, L. (2013). The surprising weak evidence base for supervision: Findings from a systematic review of research in child welfare practice (2000-2012). Children and Youth Services Review, 35, 1843-1853. doi:10.1016/j. childyouth.2013.08.014

Centore, A. J., \& Milacci, F. (2008). A study of mental health counselors' use of and perspectives on distance counselling. Journal of Mental Health Counseling, 30(3), 267-282.

Christogiorgos, S., Vassilopoulou, V., Florou, A., Xydou, V., Douvou, M., Vgenopoulou, S., \& Tsiantis, J. (2010). Telephone counselling with adolescents and countertransference phenomena: Particularities and challenges. British Journal of Guidance and Counselling, 38(3), 313-325.
Coman, G. J., Burrows, G. D., \& Evans, B. J. (2001). Telephone counselling in Australia: Applications and considerations for use. British Journal of Guidance \& Counselling, 29(2), 247-258.

Dong, C. Y. (2016). The uses of mental health telephone counselling services for Chinese speaking people in New Zealand: Demographics, presenting problems, outcome and evaluation of the calls. The New Zealand Medical Journal, 129(1441), 68-77.

Durie, M., \& Hermansson, G. (1990). Counselling Māori people in New Zealand [Aotearoa]. International Journal for the Advancement of Counselling, 14, 197-118.

Fraser, H., \& Taylor, N. (2016). Neoliberalization, Universities and the public intellectual: Species, gender and class and the production of knowledge. London, UK: Palgrave Macmillan.

Hall, B., \& Schlosar, H. (1995). Repeat callers and the Samaritan telephone crisis line: A Canadian experience. Crisis: The Journal of Crisis Intervention and Suicide Prevention, 16(2), 66-71.

Hanna, S. (2010). Call centres as sites of professional practice: "Where old social workers go to die?" Australian Social Work, 63(3), 266-280.

Hanna, S., \& Nash, M. (2012). "You don't have to shout": Vocal behaviour in social work communication. Social Work Education, 31(4), 485-497.

Harms, L. (2015). Working with people: Communication skills for reflective practice (2nd ed.). South Melbourne, VIC: Oxford University Press.

Hermansson, G. L., \& Webb, S. B. (1993). Guidance and counselling in New Zealand: Weathering a decade of transformation. International Journal for the Advancement of Counselling, 16, 213-227.

Holstein, J. A., \& Gubrium, J. F. (2004). The active interview. In D. Silverman (Ed.), Qualitative research: Theory, method and practice. London: SAGE Publications.

Holz, C. (2019). A manager's challenge: Is external supervision more valuable than increased training money for staff? Aotearoa New Zealand Social Work, 31 , 127-127-133. doi:10.11157/anzswj-vol31iss3id654

Kim, H. S., Hodkins, D. C., Bellringer, M., \& Abbott, M. (2016). Gender differences among helpline callers: Prospective study of gambling and psychosocial outcomes. Journal of Gambling Studies, 32(2), 605-623.

King, R., Nurcombe, B., Bickman, L., Hides, L., \& Reid, W (2003). Telephone counselling for adolescent suicide prevention: Changes in suicidality and mental state from beginning to end of a counselling session. Suicide and Life-Threatening Behaviour, 33(4), 400-411.

Kinzel, A., \& Nanson, J. (2000). Education and debriefing: Strategies for preventing crises in crisis-line volunteers. Crisis: The Journal of Crisis Intervention and Suicide Prevention, 21(3), 126-134.

Lang, S. K. W. (2005). "Decolonialism" and the counselling profession: The Aotearoa/New Zealand experience. International Journal for the Advancement of Counselling, 4, 557-572.

Lang, S. K. W., \& Gardiner, B. D. (2014). As they like it culture-centred counsellor education in the context of Aotearoa New Zealand: A play on bicultural pluralism. British Journal of Guidance and Counselling, 42(1), 73-85. 
Lester, D., Brockopp, G. W., \& Blum, D. (2012). The chronic caller. In D. Lester \& J. R. Rogers (Eds.), Crisis intervention and counseling by telephone and the internet (3rd ed., pp. 159-176). Springfield, Illinois: Charles C Thomas.

Lincoln, Y. S., \& Guba, E. G. (1985). Naturalistic inquiry. Beverly Hills, CA: SAGE Publications.

Maher, A., \& von Hippel, C. (2005). Individual differences in employee reactions to open-plan offices. Journal of Environmental Psychology, 25(2), 219-229.

Maxwell, J. A. (2005). Qualitative research design: An interactive approach (2nd ed.). Applied social research methods series (Vol. 41). Thousand Oaks, CA: SAGE Publications.

Miller, J. H. (2012). Counselling and guidance initiatives in Aotearoa New Zealand. British Journal of Guidance and Counselling, 40(3), 187-190

Minichiello, V., Aroni, R., \& Hays, T. (2008). In-depth Interviewing: Principles, techniques, analysis (3rd ed.). Sydney, NSW: Pearson Education Australia.

O'Sullivan, J., \& Whelan, T. A. (2011). Adversarial growth in telephone counsellors: Psychological and environmenta influences. British Journal of Guidance and Counselling, 39(4), 307-323.

Parrott, L., \& Madoc-Jones, I. (2008). Reclaiming information and communication technologies for empowering social work practice. Journal of Social Work, 8(2), 181-197.

Patton, M. Q. (2002). Qualitative research and evaluation methods. Thousand Oaks, CA: SAGE Publications.

Rhoades, L., \& Eisenberger, R. (2002). Perceived organizational support: A review of the literature. Journal of Applied Psychology, 87(4), 698-714.

Roberts, S. (2016). Assessment with Maori. In J. Maidment \& R. Egan (Eds.), Practice skills in social work and welfare: More than just common sense, (pp. 207-224). Crows Nest, NSW: Allen \& Unwin.

Rodgers, N. (2012). Shifting landscapes of counselling identities in Aotearoa New Zealand. British Journal of Guidance and Counselling, 40(3), 191-204.

Rosenfield, M. (1997). Counselling by telephone. London, UK: Sage Publications.

Rosenfield, M. (2002). Electronic technology for social work education and practice: The application of telephone technology to counseling. Journal of Technology in Human Services, 20(1-2), 173-181.

Rosenfield, M. (2003). Telephone counselling and psychotherapy in practice. In S. Goss \& K. Anthony (Eds.), Techonology in counselling and psychotherapy: A practitioner's guide (pp. 93-108). Basingstoke, UK: Palgrave Macmillan.

Ryan, G. W., \& Bernard, H. R. (2003). Techniques to identify themes. Field Methods, 15(1), 85-109. doi:10.1177/1525822X02239569

Sanders, P. (2007). Using counselling skills on the telephone and in computer-mediated communication: An incomplete guide to using counselling skills on the telephone (3rd ed.). Ross-on-Wye, UK: PCCS Books.

Sharah, S. (1995). Future directions in telephone counselling: The belief in self-control and the "illusion" of a meaningful world (Unpublished Bachelor of Art (Honours) thesis), Macquarie University, Sydney, NSW.
Shier, M. L., \& Graham, J. R. (2011). Work-related factors that impact social work practitioner's subjective wellbeing: Well-being in the workplace. Journal of Social Work, 11(4), 402-421.

Stake, R. E. (2005). Qualitative case studies. In N. K. Denzin \& Y. S. Lincoln (Eds.), The Sage handbook of qualitative research ( $3^{\text {rd }}$ ed., pp. 443-466). Thousand Oaks, CA: SAGE Publications.

Staniforth, B. (2010). Counselling in social work in Aotearoa New Zealand: The historical, political and socio-cultural evolution. Aotearoa New Zealand Social Work, 22(3), 3-13.

Stanley, P., \& Manthei, R. (2004). Counselling psychology in New Zealand: The quest for identity and recognition. Counselling Psychology Quarterly, 17(3), 301-315.

Suler, J. (2004). The online disinhibition effect. Cyberpsychology \& Behavior, 7(3), 321-326.

Van den Broek, D. (2008). "Doing things right", or "doing the right things"? Call centre migrations and dimensions of knowledge. Work, Employment \& Society, 22(4), 601-613. doi:10.1177/0950017008096738.

Watson, A. (2019). Collision: An opportunity for growth? Māori social workers' collision of their personal, progessional, and cultural worlds and the values and ethical challenges within this experience. Journal of Social Work Values and Ethics. 16(2), 28-40.

Youthline. (2005). Evidence of the effectiveness of telephone counselling services. Auckland, New Zealand: Author. 